\title{
Microlocal Quasianalyticity for Distributions and Ultradistributions
}

By

\author{
Jan BOMAN*
}

\section{§1. Introduction}

Let $S$ be a real analytic submanifold of $\boldsymbol{R}^{d}$ and let $f$ be a distribution defined in an open set $X$ containing $S$. Assume that the analy tic wave front set of $f, W F_{A}(f)$, is disjoint from the conormal bundle of $S$, i.e.

$$
W F_{A}(f) \cap N^{*}(S)=\emptyset .
$$

Then the restrictions to $S$ of $f$ and all its derivatives $\partial^{\alpha} f$ are well-defined distributions on $S$, so it makes sense to assume

$$
\left.\partial^{\alpha} f\right|_{s}=0 \text { for all } \alpha=\left(\alpha_{1}, \ldots, \alpha_{d}\right) \in N^{d}
$$

It was proved in [Bo1] that (1.1) and (1.2) imply that $f$ must vanish in some neighborhood of $S$. The purpose of this note is to strengthen that result by replacing $W F_{A}(f)$ with $W F_{M}(f)$, the wave front set of $f$ with respect to an arbitrary quasianalytic Denjoy-Carleman class $C^{M}$ (Theorem 1), and by allowing $f$ to be an ultradistribution in the dual of a non-quasianalytic class (Theorem 2).

By a counterexample of M. Sato ([Ka], Note 3.3) (1.1) and (1.2) do not imply that $f=0$ in a neighborhood of $S$, if $f$ is only assumed to be a hyperfunction. In fact we have recently proved [Bo2] that Sato's example can be strengthened as follows: for an arbitrary quasianalytic class $C^{M}$ there exists a hyperfunction in the dual of $C^{M}$ such that (1.1) and (1.2) hold for some $S$ but the support of $f$ meets $S$.

In the analytic case there is a well known closely related theorem proved by Hörmander for distributions ([H1] ; [H2], Theorem 8.5.6) and independently by Kawai and Kashiwara for hyperfunctions ([Ka], Theorem 4.4.1), which reads as follows. If the distribution $f$ vanishes on one side of the $C^{1}$ hypersurface $S$ and

Communicated by T. Kawai, May 15, 1995.

1991 Mathematics Subject Classification(s) : 35A27, 32A15, 46F15, 46F20

* Department of Mathematics, Stockholm University, S-10691 Stockholm, Sweden. 
$(x, \xi) \notin W F_{A}(f)$, where $\xi$ is one of the two conormals to $S$ at $x$, then $f$ must vanish in some neighborhood of $x$. The corresponding statement where $W F_{A}(f)$ is replaced by $W F_{M}(f), C^{M}$ quasianalytic, has also been proved by Hörmander [H4].

The special case of Theorem 2 where $f$ satisfies (1.1) has been considered by Tanabe and Takiguchi [TT].

Let $M=\left\{M_{n}\right\}_{n=1}^{\infty}$ be an increasing sequence of positive numbers and $X$ an open connected subset of $\boldsymbol{R}^{d}$. The class $C^{M}=C^{M}(X)$ is defined as the set of all $\varphi \in C^{\infty}(X)$ such that for each compact set $K \subset X$ there exists a constant $C$ such that

$$
\sup _{K}\left|\partial^{\alpha} \varphi\right| \leq C^{|\alpha|+1} M_{|\alpha|} \text { for all } \alpha \in N^{d}
$$

The class $C^{M}$ is called quasianalytic, if no non-trivial function in $C^{M}$ vanishes together with all its derivatives at any point. According to the Denjoy-Carleman Theorem the class $C^{M}$ is quasianalytic if and only if $\Sigma \bar{M}_{n} / \bar{M}_{n+1}=\infty$, or equivalently, $\sum \bar{M}_{n}^{-1 / n}=\infty$, where $\bar{M}_{n}$ is the largest logarithmically convex minorant of $M_{n}$. A class $C^{M}\left(\boldsymbol{R}^{d}\right)$ can always be defined by a logarithmically convex sequence $M_{n}$. Let $M_{n}$ be a logarithmically convex sequence such that

$$
M_{0}=1, \quad M_{n} \geq n !, \quad \text { and } \quad M_{n+1} \leq C^{n+1} M_{n}
$$

for some $C$. Those conditions ensure that $C^{M}$ is closed under multiplication and differentiation and contains the analytic class. The set of Schwartz distributions in $X$ is denoted $\mathscr{D}^{\prime}(X)$ and the set of distributions with compact support in $X$ is denoted $\mathscr{E}^{\prime}(X)$. The wave front set with respect to $C^{M}$ for $f$, denoted $W F_{M}(f)$, introduced by Hörmander in [H1], is defined as follows. The Fourier transform of $f$ is denoted $\hat{f}$.

Definition. $W F_{M}(f)$ is defined as the complement with respect to $X \times\left(\boldsymbol{R}^{d} \backslash 0\right)$ of the set of $\left(x^{0}, \xi^{0}\right)$ such that there exists a neighborhood $V \subset X$ of $x^{0}$, a conic neighborhood $\Gamma$ of $\xi^{0}$, and a bounded sequence $f_{n} \in \mathscr{E}^{\prime}(X)$, where $f_{n}=f$ in $V$ and

$$
\left|\hat{f}_{n}(\xi)\right| \leq C^{n+1} M_{n} /|\xi|^{n} \text { for } n=0,1, \ldots \text { and all } \xi \in \Gamma
$$

The requirement that the sequence $f_{n}$ be bounded is equivalent to the existence of constants $C$ and $q$ such that

$$
\left|\hat{f}_{n}(\xi)\right| \leq C(1+|\xi|)^{q}
$$

for all $n$ (cf. [H2], Prop. 8.4.2).

Theorem 1. Let $M_{n}$ be a logarithmically convex sequence satisfying (1.3), and 
assume that the class $C^{M}$ is quasianalytic. Let $f$ be a distribution defined in some neighborhood of the real analytic submanifold $S \subset \boldsymbol{R}^{d}$. Assume that the wave front set with respect to $C^{M}$ for $f, W F_{M}(f)$, is disjoint from the conormal bundle of $S$, that is,

$$
W F_{M}(f) \cap N^{*}(S)=\emptyset,
$$

and that (1.2) holds. Then $f=0$ in some neighborhood of $S$.

The more general statement where $f$ is allowed to be an ultradistribution is proved in Section 7 (Theorem 2).

Note that the condition (1.2) makes sense in Theorem 1 , since $W F_{M}(f)$ is obviously contained in $W F(f)$, the wave front set with respect to $C^{\infty}$, and $W F(f) \cap N^{*}(S)=\emptyset$ is sufficient for the restrictions $\left.\partial^{\alpha} f\right|_{S}$ to be well-defined.

Here is an outline of the proof of the theorem. To simplify the notation a little we shall first assume that $S$ is a hypersurface. The assumptions of the theorem are preserved under a real analytic change of coordinates, so we may assume that $S$ is contained in the plane $x_{d}=0$. We write $x=\left(x^{\prime}, x_{d}\right)$. The problem is local, so it is enough to study a neighborhood of one point, for instance the origin. As in [Bo1] we shall construct a sequence of cut-off functions $\chi_{n}(x)=\chi_{n}\left(x^{\prime}, x_{d}\right)$, whose derivatives of order $\leq n$ satisfy good estimates, and study the functions

$$
w_{n}\left(x_{d}\right)=\int \chi_{n}\left(x^{\prime}, x_{d}\right) f\left(x^{\prime}, x_{d}\right) d x^{\prime}
$$

The sequence $\chi_{n}$ can be chosen to tend to an arbitrary test function, so it will be sufficient to prove that $w_{n}\left(x_{d}\right)$ tends to zero as $n \rightarrow \infty$ for sufficiently small $x_{d}$. The assumption (1.2) implies that all derivatives of $w_{n}$ vanish at the origin, and (1.4) implies that the derivatives $w_{n}^{(s)}$ satisfy good estimates for $s \leq n$, for $s$ close to $n$ essentially $\left|w_{n}^{(s)}\right| \leq C^{s} M_{s}$. If, as in [Bo1], $C^{M}$ is the analytic class, we can use Taylor's formula at this point to conclude $\left|w_{n}\left(x_{d}\right)\right| \leq\left(C\left|x_{d}\right|\right)^{n}$, which implies $w_{n} \rightarrow 0$ for $\left|x_{d}\right|<1 / C$. Here we are interested in the case when $M_{n}^{1 / n} / n$ tends to infinity; we must therefore replace the remainder estimate in Taylor's formula by an estimate which takes into account bounds for derivatives $\left|w_{n}^{(s)}\right|$ of orders less than $n$ also. However, the derivatives $\partial^{\alpha} \chi_{n}$ satisfy rather bad estimates for $|\alpha| / n$ small, so our estimate for $\left|w_{n}^{(s)}\right|$ will be bad for $s / n$ small. Our substitute for Taylor's formula will therefore be an estimate for $\left|w_{n}\left(x_{d}\right)\right|$ in terms of bounds for $\left|w_{n}^{(s)}\right|$ for $s$ in some suitable interval $m \leq s \leq n$. For the same reason special care is needed in the construction of $\chi_{n}$.

\section{§2. An inequality for Flat Functions}

The following lemma and its proof are based on Bang's ideas [Ba] as simplified by Cohen [C]. The lemma can also be deduced from Lemma 1.3.6 in [H2]; see 
Remark at the end of this section. The special case $m=1$ proves of course that the class $C^{M}$ is quasianalytic if $\sum \bar{M}_{k} / \bar{M}_{k+1}$ is divergent. However, for a given finite sequence $B_{0}, \ldots, B_{n}$ (for example $B_{k}=n^{k}$ for $0 \leq k \leq n$ ) and sufficiently small $t$, larger values of $m$ often give better estimate for $u(t)$. We shall only need the case when $B_{k}$ is a logarithmically convex sequence, but we treat the general case, since it requires very little additional effort.

Lemma 1. Let $u(t)$ be a $C^{\infty}$ function on $\boldsymbol{R}$, vanishing for $t \leq 0$, let $B_{k}$, $0 \leq k \leq n$, be a sequence of positive numbers, and assume sup $\left|u^{(k)}\right| \leq B_{k}$ for $0 \leq k \leq n$. Denote by $\bar{B}_{k}$ the largest logarithmically convex minorant of $B_{k}$. Then

$$
|u(t)| \leq 2 B_{0}\left(\frac{2 t}{\sum_{m}^{n} \bar{B}_{k-1} / \bar{B}_{k}}\right)^{m}
$$

provided the expression within brackets is $\leq 1 / 2$.

Proof. We first consider the special case when $B_{k}$ is logarithmically convex; we shall prove that $u(t)$ then satisfies (2.1) with the factor 2 omitted, i.e.

$$
|u(t)| \leq B_{0}\left(\frac{2 t}{\sum_{m}^{n} B_{k-1} / B_{k}}\right)^{m}
$$

provided the expression within brackets is $\leq 1 / 2$. Consider first the case $m=1$. Let $t_{1}>0$ and divide the interval $\left[0, t_{1}\right]$ into $n$ subintervals, $I_{1}, I_{2}, \ldots, I_{n}$, numbered from right to left, so that the length of $I_{k}$ is proportional to $B_{k-1} / B_{k}$, that is, $\left|I_{k}\right|=a B_{k-1} / B_{k}$, where $a=t_{1} / \sum_{1}^{n} B_{k-1} / B_{k}$. Writing

$$
F(s, k)=\sup _{I_{k}}\left|u^{(s)}\right|
$$

we shall prove the estimate

$$
F(s, k) \leq(2 a)^{k-s} B_{s} \text { for } 0 \leq s \leq k \leq n
$$

The proof will be carried out by double induction with ascending $k$ and $s$. By assumption the estimate (2.2) is true for $k=s$, and if we set $F(s, n+1)=0$ for all $s$, (2.2) will be true for $k=n+1$ also. For the induction step we use the trivial estimate

$$
F(s, k) \leq F(s, k+1)+\left|I_{k}\right| F(s+1, k)
$$

Let $0 \leq s<k \leq n$ and assume (2.2) is true for $(s, k+1)$ and $(s+1, k)$. Then by (2.3) 


$$
F(s, k) \leq(2 a)^{k+1-s} B_{s}+\frac{a B_{k-1}}{B_{k}}(2 a)^{k-s-1} B_{s+1} .
$$

The logarithmic convexity implies that $B_{k-1} / B_{k}$ is decreasing. Hence

$$
\frac{B_{k-1}}{B_{k}} \leq \frac{B_{s}}{B_{s+1}}
$$

so that (2.4) gives

$$
F(s, k) \leq(2 a)^{k-s} B_{s}\left(2 a+\frac{a}{2 a}\right) .
$$

If now $2 a \leq 1 / 2$, the induction works, and we obtain for $s=0$ with $t_{1}=t$

$$
F(0, k) \leq B_{0}(2 a)^{k} \leq B_{0}(2 a)=B_{0} \frac{2 t}{\sum_{1}^{n} B_{k-1} / B_{k}},
$$

which completes the proof of $\left(2.1^{\prime}\right)$ in the special case when $m=1$.

To prove $\left(2.1^{\prime}\right)$ for arbitrary $m$ we restrict all considerations to the intervals $I_{k}$ with $k \geq m$. If we choose

$$
a=t / \sum_{m}^{n} B_{k-1} / B_{k}
$$

we obtain from (2.5)

$$
|u(t)| \leq F(0, m) \leq B_{0}(2 a)^{m}
$$

which proves $\left(2.1^{\prime}\right)$.

Finally we consider the case of an arbitrary sequence $B_{k}$. Setting $F_{k}=$ $\sup \left|u^{(k)}\right|$ we know by Kolmogorov's inequality that

$$
F_{k} \leq 2 F_{m}^{(n-k) /(n-m)} F_{n}^{(k-m) /(n-m)}, \quad m<k<n
$$

This means that we can apply $\left(2.1^{\prime}\right)$ to the logarithmically convex sequence $2 \bar{B}_{k}$, and observing that $\bar{B}_{0}=B_{0}$ we then obtain (2.1).

Remark. Lemma 1 can be deduced from Lemma 1.3.6 in [H2] as follows. If $a_{j}$ has the same meaning as in that lemma, we choose $a_{j}=c B_{j-1} / B_{j}$ for $j \geq m$ and $a_{j}=a_{m}$ for $1 \leq j \leq m$, where $c$ is the constant determined by the condition $\sum a_{j}$ $=t$. Then the conclusion of the lemma implies (2.1').

\section{$\S 3$. The Cut-off Functions}

Given a compact set $K \subset \boldsymbol{R}^{d}$ and an open set $U \supset \boldsymbol{K}$ it is well known how to 
construct a sequence $\chi_{n}$ of $C^{\infty}$ functions with support contained in $U$, equal to 1 on $K$, and satisfying estimates

$$
\sup \left|\partial^{\alpha} \chi_{n}\right| \leq C(C n)^{|\alpha|}, \quad|\alpha| \leq n
$$

with $C$ independent of $n$ (see [H2], Section 8.4). In this section we shall construct, for an arbitrary logarithmically convex sequence $M_{k}$ satisfying (1.3), a sequence $\chi_{n}$ satisfying (3.6) below; this estimate is slightly weaker than (3.1) for $|\alpha|=n$, but stronger for smaller $|\alpha|$.

To motivate the construction below we will first explain why the estimate (3.1) is not sufficient for our purpose. As explained in the introduction we are going to estimate the function (1.5) using Lemma 1. It will be necessary then to have bounds $B_{k, n}$ for the derivatives $\partial^{k} w_{n}, k \leq n$, that are good enough for

$$
\sum_{k=m}^{n} B_{k-1, n} / B_{k, n} \geq c>0
$$

to hold for arbitrarily large $m$ and suitable $n$. Using a sequence $\chi_{n}$ satisfying (3.1) and assuming $f$ satisfies (1.4) we would be able to prove $\left|\partial^{k} w_{n}\right| \leq C^{k+1} B_{k, n}$ for $k \leq n$, where $B_{k, n}=\max \left(n^{k}, M_{k}\right)$, but no better, since those are the estimates valid for a product $\chi_{n} f$ where $f \in C^{M}$. And it is easy to see that (3.2) does not hold then, if $M_{k}=(k \log k)^{k}$. In fact, if $k_{0}$ is the largest integer $k$ such that $k \log k<n$, then $k_{0}=(n / \log n)(1+o(1))$ as $n \rightarrow \infty$, and

$$
\begin{aligned}
\sum_{1}^{n} \frac{B_{k-1, n}}{B_{k, n}} & \leq \frac{k_{0}}{n}+\sum_{k_{0}}^{n} \frac{1}{k \log k} \leq \\
& \frac{C}{\log n}+\log \frac{\log n}{\log k_{0}}=\log \frac{\log n}{\log n-\log \log n}+o(1) \rightarrow 0 \text { as } n \rightarrow \infty,
\end{aligned}
$$

which contradicts (3.2).

Take a non-negative, even function $v \in C^{\infty}$ with support in $(-1,1)$ and with integral equal to 1 . It is clearly possible to choose $v$ such that $\int ! v^{\prime}(t) \mid d t \leq 2$. For $0<\varepsilon<1$ take

$$
\phi(x)=(\sqrt{d} / \varepsilon)^{d} \prod_{\nu=1}^{d} v\left(x_{\nu} \sqrt{d} / \varepsilon\right) .
$$

Then $\phi$ is non-negative, even, $\phi \in C^{\infty}$, the support of $\phi$ is contained in an $\varepsilon$ neighbourhood of the origin, $U_{\varepsilon}$, and

$$
\int\left|\partial_{\nu} \phi(x)\right| d x=\frac{\sqrt{d}}{\varepsilon} \int\left|v^{\prime}(t)\right| d t \leq \frac{2 \sqrt{d}}{\varepsilon}, \quad 1 \leq \nu \leq d .
$$

Let $\theta_{n}$ be the convolution 


$$
\theta_{n}(x)=\frac{1}{a_{1}} \phi\left(\frac{x}{a_{1}}\right) * \ldots * \frac{1}{a_{n}} \phi\left(\frac{x}{a_{n}}\right),
$$

where $a_{1}, \ldots, a_{n}$ are positive numbers with $\sum a_{k}=1$. For the constants $a_{k}$ we make the choice

$$
a_{k}=a_{k}(n)=\frac{1}{s_{n}} \cdot \frac{M_{k-1}}{M_{k}}
$$

where

$$
s_{n}=\sum_{k=1}^{n} M_{k-1} / M_{k} \text {. }
$$

Then supp $\theta_{n} \subset U_{\varepsilon}, \int \theta_{n} d x=1$, and for $|\beta| \leq n$ we can compute $\partial^{\beta} \theta_{n}$ by applying a first order partial derivative to each of the first $|\beta|$ factors in (3.4), hence

$$
\int ! \partial^{\beta} \theta_{n} \mid d x \leq B s_{n} \frac{M_{1}}{M_{0}} \cdot \ldots \cdot B s_{n} \frac{M_{|\beta|}}{M_{|\beta|-1}}=\left(B s_{n}\right)^{|\beta|} M_{|\beta|} \cdot
$$

Here the constant $B$ is the number $(2 \sqrt{d}) / \varepsilon$ appearing in (3.3). For $\varphi \in C_{0}^{\infty}\left(\boldsymbol{R}^{n}\right)$ we choose

$$
\chi_{n}=\varphi * \theta_{n} .
$$

Then

$$
\left|\partial^{\alpha+\beta} \chi_{n}\right|=\left|\partial^{\alpha} \varphi * \partial^{\beta} \theta_{n}\right| \leq \sup \left|\partial^{\alpha} \varphi\right| \int\left|\partial^{\beta} \theta_{n}\right| d x \leq C_{\alpha}\left(B s_{n}\right)^{|\beta|} M_{|\beta|},|\beta| \leq n .
$$

We next prove that $\chi_{n} \rightarrow \varphi$ in $C_{0}^{\infty}$. The support of $\chi_{n}$ is contained in an $\varepsilon$ neighborhood of the support of $\varphi$, and for an arbitrary derivative we have the estimate

$$
\left|\partial^{\alpha} \chi_{n}-\partial^{\alpha} \varphi\right|=\left|\theta_{n} * \partial^{\alpha} \varphi-\partial^{\alpha} \varphi\right| \leq \int\left|\left(\hat{\theta}_{n}(\xi)-1\right) \widehat{\partial^{\alpha} \varphi}(\xi)\right| d \xi
$$

Since $\hat{\theta}_{n}$ is bounded, it is therefore sufficient to verify that $\hat{\theta}_{n}(\xi)$ tends to 1 uniformly on compact sets. To do this, note that $\hat{\phi}^{\prime}(0)=0$ since $\phi$ is even, and hence

$$
\left|\log \hat{\theta}_{n}(\xi)\right|=\left|\sum_{k=1}^{n} \log \hat{\phi}\left(a_{k} \xi\right)\right| \leq C \sum_{k=1}^{n}\left|a_{k} \xi\right|^{2} \leq C_{1} \sum_{k=1}^{n} a_{k}^{2}
$$

for $\xi$ in a compact set. Since $a_{k}$ is decreasing and $\Sigma a_{k}=1$ we have 


$$
\sum_{1}^{n} a_{k}^{2} \leq a_{1} \sum_{1}^{n} a_{k}=a_{1}=\frac{M_{0}}{M_{1} s_{n}} \rightarrow 0, \text { as } n \rightarrow \infty,
$$

which proves the claim.

If $\varphi=1$ in some neighborhood of the compact set $K$ and $\varepsilon$ is sufficiently small, then also $\chi_{n}=1$ in some fixed neighborhood of $K$. This justifies the term cut-off functions for $\chi_{n}$.

It is natural to ask for which sequences $B_{k, n}$ with $B_{0, n}=1$ for all $n$ there exists a sequence $\chi_{n}$ as above satisfying

$$
\sup \left|\partial^{\alpha} \chi_{n}\right| \leq C^{k+1} B_{k, n} \text { for }|\alpha|=k \leq n, n=0,1, \ldots
$$

It is easy to see that the construction above works provided

$$
\sup _{n} \sum_{k=1}^{n} \bar{B}_{k-1, n} / \bar{B}_{k, n}<\infty \text {; }
$$

here $k \mapsto \bar{B}_{k, n}$ is the largest logarithmically convex minorant of $k \mapsto B_{k, n}$. On the other hand, if (3.8) does not hold and $\chi_{n} \in C^{\infty}(\boldsymbol{R})$ is supported in a fixed bounded interval and satisfies (3.7), then Lemma 1 with $m=1$ shows that some subsequence of $\chi_{n}$ must tend uniformly to zero. Taking $B_{k, n}=k$ ! for $k \leq n$ and all $n$ we see in particular that cut-off functions satisfying the stronger estimates

$$
\sup \left|\partial^{\alpha} \chi_{n}\right| \leq C^{k+1} k !,|\alpha|=k \leq n
$$

do not exist.

Repeated convolutions (3.4) with dilation factors $a_{k}$ proportional to $M_{k-1} / M_{k}$ were used by Cohen [C] in his proof of the Denjoy-Carleman Theorem; in fact, if $\sum M_{k-1} / M_{k}<\infty$, then $\theta_{n}$ as given by (3.4) converges as $n \rightarrow \infty$ to a non-trivial function in $C^{M}$ with compact support.

\section{§4. Estimates for $\widehat{\chi_{n} f}$}

Assume $f$ satisfies the assumptions of the theorem and $S$ is an open subset of the hyperplane $x_{d}=0$. Let $x^{0} \in S$. The definition of the wave front set with respect to $C^{M}$ then means that there exists a conic open neighborhood $\Gamma$ of $\pm d x_{d}=(0, \ldots, 0, \pm 1)$ and distributions $f_{n}, n=1,2, \ldots$, with compact support, equal to $f$ in a fixed open neighborhood $V$ of $x^{0}$, and constants $C$ and $A_{0}$ such that

$$
\left|\hat{f}_{n}(\xi)\right| \leq C\left(A_{0} /|\xi|\right)^{n} M_{n} \text { for all } \xi \in \Gamma \text { and all } n
$$

and, if $f$ has order $q$, 


$$
\left|\hat{f}_{n}(\xi)\right| \leq C(1+|\xi|)^{q} \text { for all } n
$$

Choose a number $b, 0<b<1 / 2$, and a conic neighborhood $\Gamma_{1}$ of $\pm d x_{d}$ such that

$$
\xi \in \Gamma_{1} \text { and }|\eta|<b|\xi| \text { implies } \xi+\eta \in \Gamma .
$$

Let $\varphi \in C_{0}^{\infty}(V)$, choose $\varepsilon, 0<\varepsilon<1$, so small that a $2 \varepsilon$-neighborhood of supp $\varphi$ is contained in $V$, let $\chi_{n}=\theta_{n} * \varphi$ be the sequence of functions constructed above, and let the constant $B$ be chosen as in Section 3. We must now estimate $\widehat{\chi_{n} f}$.

Lemma 2. Assume (4.1) and (4.2) hold, and let $\varepsilon, B, b, \Gamma_{1}$, and $\chi_{n}$ be chosen as described above. Then

$$
\left|\widehat{\chi_{n} f}(\xi)\right| \leq C \frac{A^{k}\left(1+s_{n}\right)^{k}}{(1+|\xi|)^{k}} M_{k} \text { for all } \xi \in \Gamma_{1} \text { and all } k \leq n
$$

with $A=2 \max \left\{2 A_{0}, B / b\right\}$; here $s_{n}$ is defined by (3.5). The constant $C$ is independent of $k$ and $n$, but may depend on $\varphi$.

Proof. As in [Bo1] we write

$$
\begin{aligned}
& \left|\widehat{\chi_{n} f}(\xi)\right|=\left|\widehat{\chi}_{n} f_{k}(\xi)\right|=\left|\int \hat{\chi}_{n}(\eta) \hat{f}_{k}(\xi-\eta) d \eta\right| \leq \sup _{|\eta|<b|\xi|}\left|\hat{f}_{k}(\xi-\eta)\right| \int\left|\hat{\chi}_{n}(\eta)\right| d \eta \\
& \quad+\sup _{|\eta|>b|\xi|}(1+|\eta|)^{-q}\left|\hat{f}_{k}(\xi-\eta)\right| \int_{|\eta|>b|\xi|}\left|\hat{\chi}_{n}(\eta)\right|(1+|\eta|)^{q} d \eta=I+I I .
\end{aligned}
$$

We first estimate the term $I$. From (3.6) with $|\beta|=0$ and $|\alpha|=s$ we get

$$
\left|\hat{\chi}_{n}(\xi)\right| \leq C_{s}(1+|\xi|)^{-s}
$$

and with $s=d+1$ here we see that $\int\left|\hat{\chi}_{n}(\eta)\right| d \eta \leq C$. Here and below the letter $C$ will denote constants that are independent of $n$ but may depend on $f$ and on the function $\varphi$ which was used in the construction of the sequence $\chi_{n}$. Noting that $|\xi-\eta| \geq|\xi| / 2$ when $|\eta|<b|\xi|$ we see that (4.1) implies

$$
I \leq C\left(\frac{2 A_{0}}{|\xi|}\right)^{k} M_{k} \leq C\left(\frac{4 A_{0}}{1+|\xi|}\right)^{k} M_{k} \text { for all } \xi \in \Gamma_{1},|\xi|>1, k \geq 0 .
$$

For $|\xi|<1$ we can use (4.2) and get $I \leq C \leq C 2^{k}(1+|\xi|)^{-k}$; hence $I$ is bounded by the right hand side of (4.3) if $A=\max \left\{4 A_{0}, 2\right\}$ (note that $B / b>1$ ).

We now estimate the term $I I$. With $s=q+d+1$ in (4.5) and using (4.2) we see that

$$
I I \leq C \text { for all } \xi \text {. }
$$


Taking $|\alpha|=q+d+1$ and $|\beta|=k$ we can also deduce from (3.6) that

$$
\left|\hat{\chi}_{n}(\xi)\right| \leq C(1+|\xi|)^{-q}|\xi|^{-d-1-k}\left(B s_{n}\right)^{k} M_{k} \text { for } k \leq n
$$

this leads to

$$
\begin{aligned}
I I \leq C\left(B s_{n}\right)^{k} M_{k} \int_{|\eta|>b|\xi|} \frac{d \eta}{|\eta|^{d+1+k}}=C\left(\frac{B s_{n}}{b|\xi|}\right)^{k} M_{k} \int_{|\eta|>1} \frac{d \eta}{|\eta|^{d+1+k}} \\
\quad \leq C_{1}\left(\frac{B s_{n}}{b|\xi|}\right)^{k} M_{k} \text { for all } \xi \text { and all } k \leq n
\end{aligned}
$$

Using this estimate for $|\xi|>1$ and (4.6) for $|\xi|<1$ we now easily see that II is bounded by the right hand side of (4.3) for all $\xi$ and $k \leq n$. This completes the proof of the lemma.

\section{§ 5. Estimates for $w_{n}$}

Let $\pi$ be the projection $\left(x^{\prime}, x_{d}\right) \mapsto x_{d}$ from $\boldsymbol{R}^{d}$ to $\boldsymbol{R}$, and let $g$ be a distribution on $\boldsymbol{R}^{d}$ such that the restriction of $\pi$ to the support of $g$ is proper. Then the pushforward $\pi_{*} g$ is a distribution on $\boldsymbol{R}$ defined by $\langle\pi * g, \varphi\rangle=\langle g, \varphi \circ \pi\rangle$ for $\varphi \in$ $C_{0}^{\infty}(\boldsymbol{R})$. Here $\langle\cdot, \cdot\rangle$ is the pairing between distributions and test functions. We now define $w_{n}$ as $\pi_{*}\left(\chi_{n} f\right)$; this is the precise meaning of (1.5). Since $f$ satisfies (1.4), $w_{n}$ must be $C^{\infty}$, and since $f$ is flat along $S=\left\{x_{d}=0\right\}, w_{n}$ must be flat at the origin. Restricting to $\xi^{\prime}=0$ in (4.3) we obtain

$$
\left|\hat{w}_{n}\left(\xi_{d}\right)\right|=\left|\widehat{\chi_{n} f}\left(0, \xi_{d}\right)\right| \leq C \frac{A^{k}\left(1+s_{n}\right)^{k}}{\left(1+\left|\xi_{d}\right|\right)^{k}} M_{k}, \quad k \leq n .
$$

Hence

$$
\sup \left|w_{n}^{(k)}\right| \leq \int\left|\tau^{k} \hat{w}_{n}(\tau)\right| d \tau \leq C_{1} A^{k+2}\left(1+s_{n}\right)^{k+2} M_{k+2}, \quad k \leq n-2
$$

With $B_{k}=B_{k, n}=C_{1} A^{k+2}\left(1+s_{n}\right)^{k+2} M_{k+2}$ we now compute a sum of the kind occurring in Lemma 1:

$$
\sum_{m-2}^{n-2} \frac{B_{k-1}}{B_{k}}=\frac{1}{A\left(1+s_{n}\right)} \sum_{m}^{n} \frac{M_{k-1}}{M_{k}}=\frac{1}{A} \cdot \frac{\sum_{m}^{n} M_{k-1} / M_{k}}{1+\sum_{1}^{n} M_{k-1} / M_{k}} .
$$

Since $\Sigma M_{k} / M_{k+1}$ is divergent, we can choose a sequence of numbers $m=m_{n}$ tending to infinity as $n \rightarrow \infty$, such that the quotient is $>1 / 2$. Applying Lemma 1 we can now conclude that $\mid w_{n}\left(x_{d}\right) ! \rightarrow 0$ as $n \rightarrow \infty$, provided $\left|x_{d}\right|<1 / 4 A$. But we have also seen that $\chi_{n} \rightarrow \varphi$ in $C_{0}^{\infty}$, hence $w_{n}\left(x_{d}\right)=\int \chi_{n} f d x^{\prime} \rightarrow \int f \varphi d x^{\prime}$ as $n \rightarrow \infty$. But $\varphi \in C_{0}^{\infty}$ was arbitrary, hence $f$ must vanish in $V \cap\left\{x ;\left|x_{d}\right|<1 / 4 A\right\}$. This completes the proof of Theorem 1 in the special case when $S$ is a hypersurface.

Assume now that $S$ is a submanifold of arbitrary dimension $r, 0 \leq r \leq d-1$. 
Let $S$ be defined by $x^{\prime \prime}=0$, where $x=\left(x^{\prime}, x^{\prime \prime}\right), x^{\prime} \in \boldsymbol{R}^{r}, x^{\prime \prime} \in \boldsymbol{R}^{d-r}$, and define $w_{n}$ by (1.5) with $x_{d}$ replaced by $x^{\prime \prime}$. Then $w_{n} \in C^{\infty}\left(\boldsymbol{R}^{d-r}\right)$, and writing $\xi=\left(\xi^{\prime}, \xi^{\prime \prime}\right)$ we have (5.1) with $\xi_{d}$ replaced by $\xi^{\prime \prime}$. Hence

$$
\left|\partial^{\alpha} w_{n}\right| \leq C_{1} A^{k}\left(1+s_{n}\right)^{k} M_{k} \text { for }|\alpha|+d-r+1 \leq k \leq n
$$

The definition of the class $C^{M}$ is obviously invariant under a rotation of the coordinate system; hence directional derivatives of $w_{n}$ of order $|\alpha|$ satisfy similar estimates. Applying the reasoning above to the restrictions of $w_{n}$ to lines through the origin in $\boldsymbol{R}^{d-r}$ with all directions therefore finishes the proof as before.

\section{§ 6. Preliminaries on Ultradistributions}

We will now briefly review the definition and basic properties of nonquasianalytic ultradistributions, that is, elements in the dual of a non-quasianalytic class $C^{L}$. Such distributions were introduced by Beurling [B] and Roumieu [Ro1], [Ro2]; Beurling's theory was worked out in detail by Björck [Bj]. Extensive studies of non-quasianalytic ultradistributions have later been undertaken by Komatsu [Ko1], [Ko2], [Ko3]. One obtains still larger spaces of distributions by considering the dual of quasianalytic classes; then one speaks about quasianalytic ultradistributions or infrahyperfunctions. Basic properties of spaces of ultradistributions, quasianalytic or not, have been established by Hörmander [H3] and de Reuver [dR]. The largest class of quasianalytic ultradistributions is formed by the hyperfunctions of Sato, which can locally be identified with linear forms on the space of real analytic functions.

Let $L_{k}$ be a logarithmically convex sequence satisfying (1.3) and assume $C^{L}$ is non-quasianalytic, that is, $\Sigma L_{k}^{-1 / k}<\infty$. Let $X$ be an open connected subset of $\boldsymbol{R}^{d}$ and denote by $\mathscr{D}_{L}(X)$ the set of functions in $C^{L}\left(\boldsymbol{R}^{d}\right)$ that have compact support in $X$. Observe that a function $\varphi \in C_{0}^{\infty}\left(\boldsymbol{R}^{d}\right)$ belongs to $C^{L}\left(\boldsymbol{R}^{d}\right)$ if and only if the norm

$$
\|\varphi\|_{L, r}=\sup _{\alpha} r^{|\alpha|} L_{|\alpha|}^{-1} \sup \left|\partial^{\alpha} \varphi\right|
$$

is finite for some $r>0$. The set $\mathscr{D}_{L}^{\prime}(X)$ is defined as the set of linear forms $f$ on $\mathscr{D}_{L}(X)$ such that for every compact set $K \subset X$ and every $r>0$ there exists a constant $C_{r, K}$ such that

$$
|f(\varphi)| \leq C_{r, K}\|\varphi\|_{L, r}
$$

for all $\varphi \in \mathscr{D}_{L}(X)$ with support in $K$. Since there exist partitions of unity in $\mathscr{D}_{L}(X)$, one can define the restriction of an ultradistribution $f$ to an open set $\Omega \subset X$ by restricting the linear form $f$ to the set of $\varphi$ with support in $\Omega$. The support of $f$ can then be defined as the complement of the largest open set on 
which $f$ is equal to zero. The set of elements in $\mathscr{D}_{L}^{\prime}(X)$ with compact support is denoted $\mathscr{E}_{L}^{\prime}(X)$, and this space can of course be identified with a subspace of $\mathscr{E}_{L}^{\prime}\left(\boldsymbol{R}^{d}\right)=\mathscr{E}_{L}^{\prime}$. For $f \in \mathscr{E}_{L}^{\prime}$ it is clear that $f(\varphi)$ can be defined in a unique way for arbitrary $\varphi \in C^{L}\left(\boldsymbol{R}^{d}\right)$.

If $B_{k} \leq L_{k}, C^{B}$ and $C^{L}$ both non-quasianalytic, then $\mathscr{D}_{B}(X)$ is dense in $\mathscr{D}_{L}(X)$, that is, if $\varphi \in \mathscr{D}_{L}(X)$, there exists $\varphi_{n} \in \mathscr{D}_{B}(X)$, such that $\left\|\varphi_{n}-\varphi\right\|_{L, r} \rightarrow 0$ for some $r$, hence $\mathscr{D}_{L}^{\prime}(X)$ is naturally identified with a subspace of $\mathscr{D}_{B}^{\prime}(X)$.

Since $C^{L}(X)$ is a ring, we can define multiplication of $\phi \in C^{L}(X)$ with $f \in$ $\mathscr{D}_{L}^{\prime}(X)$ by $(\phi f)(\varphi)=f(\phi \varphi)$ for $\varphi \in \mathscr{D}_{L}(X)$.

The classes $C^{L}$ are stable under real analytic maps as shown in [H2], Proposition 8.4.1. Let $Y$ be another open set and let $\rho$ be a proper real analy tic map $Y \rightarrow X$. The argument in the cited proposition proves also that the composition map $\varphi \mapsto \varphi \circ \rho$ from $\mathscr{D}_{L}(X)$ to $\mathscr{D}_{L}(Y)$ is continuous in the sense that

$$
\|\varphi \circ \rho\|_{L, r} \leq C\|\varphi\|_{L, A r}
$$

where $C$ and $A$ depend only on the map $\rho$ and on the support of $\varphi$. This proves that the space $\mathscr{D}_{L}^{\prime}(X)$ is invariant under real analytic coordinate transformations and that it makes sense to speak about the spaces $C^{L}(Y), \mathscr{D}_{L}^{\prime}(Y)$, etc., where $Y$ is a real analy tic submanifold of $\boldsymbol{R}^{d}$.

The Fourier transform of $\varphi \in \mathscr{D}_{L}\left(\boldsymbol{R}^{d}\right)$ satisfies an estimate

$$
|\hat{\varphi}(\xi)| \leq C_{r} / \tilde{L}(r|\xi|)
$$

for some $r>0$, where $\tilde{L}(\rho)$ is defined by

$$
\tilde{L}(\rho)=\sup _{k} \rho^{k} / L_{k}, \rho>0 .
$$

For $f \in \mathscr{E}_{L}^{\prime}$ the Fourier transform $\hat{f}$ can be defined as $\hat{f}(\xi)=f\left(e_{-\xi}\right)$, where $e_{\xi}(x)=\exp (i x \cdot \xi)$. It follows from (6.1) that $\hat{f}$ is a real analytic function (in fact entire) and satisfies the estimate

$$
|\hat{f}(\xi)| \leq C_{r} \tilde{L}(r|\xi|)
$$

for every $r>0$.

For further basic properties of ultradistributions we refer to the papers cited above.

We now need to define the standard $\left(C^{\infty}\right)$ wave front set for an ultradistribution $f \in \mathscr{D}_{L}^{\prime}(X)$ as well as the wave front set of $f$ with respect to some other class $C^{M}$, quasianalytic or not.

Definition. Let $f \in \mathscr{D}_{L}^{\prime}(X)$. Then $W F(f)$ is defined as the complement with 
respect to $X \times\left(\boldsymbol{R}^{d} \backslash 0\right)$ of the set of $\left(x^{0}, \xi^{0}\right)$ such that there exists $g \in \mathscr{E}_{L}^{\prime}(X)$ with $g=f$ in some neighborhood of $x^{0}$, a conic neighborhood $\Gamma$ of $\xi^{0}$, and constants $C_{n}$, such that

$$
|\hat{g}(\xi)| \leq C_{n} /|\xi|^{n} \text {, for } n=1,2, \ldots \text { and all } \xi \in \Gamma \text {. }
$$

If $M_{k}$ is another logarithmically convex sequence satisfying (1.3), we define $W F_{M}(f)$, the wave front set of $f$ with respect to $C^{M}$, as the complement with respect to $X \times\left(\boldsymbol{R}^{d} \backslash 0\right)$ of the set of $\left(x^{0}, \xi^{0}\right)$ such that there exists a neighborhood $V \subset X$ of $x^{0}$, a conic neighborhood $\Gamma$ of $\xi^{0}$, and a sequence $f_{n} \in \mathscr{E}_{L}^{\prime}(X)$, where $f_{n}=f$ in $V$, satisfying

$$
\left|\hat{f}_{n}(\xi)\right| \leq C^{n+1} M_{n} /|\xi|^{n} \text {, for } n=0,1, \ldots \text { and all } \xi \in \Gamma \text {, }
$$

and

$$
\left|\hat{f}_{n}(\xi)\right| \leq C \tilde{L}(r|\xi|)
$$

for some $C$ and $r$ independent of $n$.

If $f \in \mathscr{D}_{L}^{\prime}(X)$ and $\phi \in C^{L}(X)$ we have $W F(\phi f) \subset W F(f)$. Similarly, if $\phi \in C^{L}(X) \cap C^{M}(X)$, then $W F_{M}(\phi f) \subset W F_{M}(f)$. Using (6.2) and (6.3) one proves these facts in the same way as the corresponding statements for Schwartz distributions (cf. Lemma 8.1.1 and Theorem 8.4.7 in [H2]). Note that $W F(f)$ and $W F_{M}(f)$ do not depend on the sequence $\left\{L_{k}\right\}$.

For a Schwartz distribution $f$ satisfying (1.4) the restriction $\left.f\right|_{S}$ to a smooth submanifold $S$ was defined by Theorem 8.2.4 in [H2]. For hyperfunctions satisfying (1.4) the restriction to a submanifold has also been defined (see e.g. [Ka]). Ultradistributions can be imbedded in the space of hyperfunctions, so we could refer to the theory of hyperfunctions for the definition of $\left.f\right|_{S}$ for the ultradistributions considered here. However, we prefer to make a minor extension of Hörmander's construction in order to avoid relying on the theory of hyperfunctions at this point.

If $S$ is an open part of a hyperplane in $\boldsymbol{R}^{d}$, say the hyperplane $x_{d}=0$, and $f \in C_{0}^{\infty}\left(\boldsymbol{R}^{d}\right)$, then the restriction $\left.f\right|_{S}$ satisfies

$$
\left\langle\left. f\right|_{S}, \varphi\right\rangle=\int f\left(x^{\prime}, x_{d}\right) \varphi\left(x^{\prime}\right) d x^{\prime}=(2 \pi)^{-d} \int \hat{f}\left(\xi^{\prime}, \xi_{d}\right) \hat{\varphi}\left(-\xi^{\prime}\right) d \xi^{\prime} d \xi_{d}
$$

for $\varphi \in C_{0}^{\infty}(S)$. Let $f \in \mathscr{D}_{L}^{\prime}\left(\boldsymbol{R}^{d}\right)$ and assume $f$ satisfies

$$
W F(f) \cap N^{*}(S)=\emptyset .
$$

Using a partition of unity we can then write $f=f_{1}+\ldots+f_{N}+h$, where $h$ vanishes 
in a neighborhood of $S$ and each $f_{\nu}$ satisfies (6.4), which in this case means that there is an $\varepsilon>0$ and constants $C_{n}$ such that

$$
\left|\hat{f}_{\nu}\left(\xi^{\prime}, \xi_{d}\right)\right| \leq C_{n} /\left|\xi_{d}\right|^{n}, n=1,2, \ldots
$$

provided $\left|\xi^{\prime}\right|<\varepsilon\left|\xi_{d}\right|$. Then the integral on the right hand side of (6.6) makes sense for $f_{\nu}$, so we can take this as our definition of $\left\langle\left. f_{\nu}\right|_{S}, \varphi\right\rangle$ and define $\left.f\right|_{S}=$ $\left.\sum f_{\nu}\right|_{S}$. We need to check that this definition has the right invariance properties and extend it to arbitrary analytic surfaces $S$. Following Hörmander ([H2], Section 8.2), for a closed subset $E \subset T^{*}(X)$, conic in the cotangent variable, we denote by $\mathscr{D}_{L, E}^{\prime}(X)$ the set of $f \in \mathscr{D}_{L}^{\prime}(X)$ such that $W F(f) \subset E$, and say that $f_{j} \rightarrow f$ in $\mathscr{D}_{L, E}^{\prime}(X)$ if $f_{j} \rightarrow f$ in $\mathscr{D}_{L}^{\prime}(X)$ (weakly) and in addition

$$
\sup _{F}|\xi|^{j} \mid \widehat{\phi f}_{j}(\xi)-\widehat{\phi f}(\xi) ! \rightarrow 0 \text {, as } j \rightarrow \infty \text {, }
$$

for every closed cone $F$ in $\boldsymbol{R}^{d}$ such that $\operatorname{supp} \phi \times F \cap E=\emptyset$. Now, if $S$ is contained in the plane $x_{d}=0$, and $\left.f\right|_{S}$ is defined as above, it is easy to see that the map $\rho_{S}:\left.f \mapsto f\right|_{S}$ is continuous from $\mathscr{D}_{L, E}^{\prime}(X)$ into $\mathscr{D}_{L}^{\prime}(S)$, and that this is the unique extension of $\rho_{S}$ from $C_{0}^{\infty}\left(\boldsymbol{R}^{d}\right)$ to $\mathscr{D}_{L, E}^{\prime}(X)$ with the continuity property just described. This shows that $\rho_{S}$, originally defined on $C^{\infty}(X)$, has a unique continuous extension to $\mathscr{D}_{L, E}^{\prime}(X)$ also for an arbitrary analytic hypersurface $S$, and this defines $\left.f\right|_{S}$ in the general case. The same reasoning can of course be applied if $S$ is an analytic surface with codimension greater than 1 .

Let $g \in \mathscr{C}_{L}^{\prime}\left(\boldsymbol{R}^{d}\right)$, let $S$ be the plane $x_{d}=0$ and assume $g$ satisfies (6.7). Let $\pi$ be the projection $\left(x^{\prime}, x_{d}\right) \mapsto x_{d}$ as in Section 5. For the proof of Theorem 2 we need to verify that $w\left(x_{d}\right)=\left(\pi_{*} g\right)\left(x_{d}\right)$ must be flat at the origin if $g$ is flat at $S$. (A distribution satisfying (1.2) is said to be flat at $S$.) By the definition of $w$ we have $\hat{w}(\tau)=\hat{g}(0, \tau)$, hence $w \in C^{\infty}$ by virtue of $(6.7)$ and $w^{(k)}(0)=$ $(1 / 2 \pi) \int \tau^{k} \hat{g}(0, \tau) d \tau$. On the other hand, the definition of restriction shows that the Fourier transform of $\left.\left(\partial_{d}^{k} g\right)\right|_{s}$ is

$$
\frac{1}{2 \pi} \int\left(i \xi_{d}\right)^{k} \hat{g}\left(\xi^{\prime}, \xi_{d}\right) d \xi_{d} .
$$

Now, the fact that $g$ is flat at $S$ implies that this expression vanishes for all $\xi^{\prime}$, in particular for $\xi^{\prime}=0$, hence $w^{(k)}(0)=0$.

The verification that $\chi f$ is flat along $S$ if $\chi \in \mathscr{D}_{L}\left(\boldsymbol{R}^{d}\right)$ and $f \in \mathscr{E}_{L}^{\prime}\left(\boldsymbol{R}^{d}\right)$ is flat along $S$ is even easier (see [Bol], p. 1234).

\section{§ 7. A Vanishing Theorem for Ultradistributions}

We can now state our extension of Theorem 1 to ultradistributions associated to a non-quasianaly tic class. 
Theorem 2. Let $L_{n}$ and $M_{n}$ be logarithmically convex sequences satisfying (1.3) such that $M_{n} \leq L_{n}$. Assume that the class $C^{L}$ is non-quasianalytic and that $C^{M}$ is quasianalytic. Let $f \in \mathscr{D}_{L}^{\prime}(X)$ be an ultradistribution defined in some neighborhood $X$ of the real analytic submanifold $S \subset \boldsymbol{R}^{d}$. Assume that the wave front set with respect to $C^{M}$ for $f, W F_{M}(f)$, is disjoint from the conormal bundle of $S$, that is, that (1.4) holds. Assume furthermore that $f$ is flat along $S$ in the sense that (1.2) holds. Then $f=0$ in some neighborhood of $S$.

The proof of Theorem 2 is essentially parallel to that of Theorem 1; to avoid repetitions we will therefore describe only those parts of the proof that require modifications of the proof given above.

For the cut-off functions $\chi_{n}$ we take $\phi \in \mathscr{D}_{L}\left(\boldsymbol{R}^{d}\right)$, construct $\theta_{n}$ with (3.6) as before, and finally set $\chi_{n}=\varphi * \theta_{n}$, where $\varphi \in \mathscr{D}_{L}\left(\boldsymbol{R}^{d}\right)$. For the proof of an improvement of the estimate (3.6) to be discussed below we shall in fact need to take $\varphi$ such that $\|\phi\|_{L, r}<\infty$ for every $r>0$. That such functions $\varphi$ exist is easily seen from the fact that for every logarithmically convex sequence $L_{k}$ such that $\Sigma L_{k}^{-1 / k}<\infty$ there exists another logarithmically convex sequence $B_{k}$ such that $\lim L_{k}^{-1 / k} / B_{k}^{-1 / k}=0$ and $\Sigma B_{k}^{-1 / k}<\infty$, and we can take $\varphi \in \mathscr{D}_{B}\left(\boldsymbol{R}^{d}\right)$. We need to prove first of all that $\left\|\chi_{n}-\varphi\right\|_{L, r} \rightarrow 0$ for some $r>0$ (in fact true for any $r>0$ ), that is

$$
\sup _{\alpha} r^{|\alpha|} L_{|\alpha|}^{-1} \sup \left|\partial^{\alpha}\left(\chi_{n}-\varphi\right)\right| \rightarrow 0 \text { as } n \rightarrow \infty .
$$

Since $\varphi \in \mathscr{D}_{L}\left(\boldsymbol{R}^{d}\right)$, we have sup $\left|\partial^{\alpha} \varphi\right| \leq C^{|\alpha|+1} L_{|\alpha|}$ for some $C$, hence

$$
\left|\partial^{\alpha}\left(\chi_{n}-\varphi\right)\right| \leq\left|\theta_{n} * \partial^{\alpha} \varphi\right|+\left|\partial^{\alpha} \varphi\right| \leq 2 C^{|\alpha|+1} L_{|\alpha|} \mid
$$

If we take $r \leq 1 / 2 C$, this means that

$$
r^{|\alpha|} L_{|\alpha|}^{-1} \sup \left|\partial^{\alpha}\left(\chi_{n}-\varphi\right)\right| \leq 2 C \cdot 2^{-|\alpha|}
$$

for all $\alpha$. It remains therefore only to recall that $\partial^{\alpha}\left(\chi_{n}-\varphi\right)$ tends to zero as $n \rightarrow \infty$ for each fixed $\alpha$, which was proved in Section 3 .

In estimating $\widehat{\chi_{n} f}(\xi)$ we shall need an improved version of (3.6). By the choices of $\varphi$ and $\phi$ we obtain as in (3.6)

$$
\left|\xi^{\alpha+\beta} \hat{\chi}_{n}(\xi)\right| \leq C_{r} L_{|\alpha|} r^{-|\alpha|}\left(B s_{n}\right)^{|\beta|} M_{|\beta|}, \quad|\beta| \leq n,
$$

or, with a new $C_{r}$,

$$
(r|\xi|)^{k} L_{k}^{-1}\left|\hat{\chi}_{n}(\xi)\right| \leq C_{r}\left(B s_{n} /|\xi|\right)^{j} M_{\jmath}, \quad j \leq n,
$$


for arbitrarily large $r$. Replacing $k$ by $k+d+1$ and using the fact that $L_{k}$ is increasing we obtain with still a new $C_{r}$

$$
|\xi|^{d+1}(r|\xi|)^{k} L_{k}^{-1}\left|\hat{\chi}_{n}(\xi)\right| \leq C_{r}\left(B s_{n} /|\xi|\right)^{j} M_{j}, \quad j \leq n
$$

Taking supremum over $k$ we finally obtain

$$
\left|\hat{\chi}_{n}(\xi)\right| \leq C_{r}|\xi|^{-d-1} \tilde{L}(r|\xi|)^{-1}\left(B s_{n} /|\xi|\right)^{j} M_{j}, \quad j \leq n
$$

Lemma 2 is valid for $f \in \mathscr{D}_{L}^{\prime}\left(\boldsymbol{R}^{d}\right)$ with the only change that the assumption (4.2) has to be replaced by (6.5), which means that $f_{n}$ is a bounded sequence in $\mathscr{C}_{L}^{\prime}$. In the proof of Lemma 2 the term $I$ in (4.4) can be treated as before. In estimating the term $I I$ we need to replace the factor $(1+|\eta|)^{q}$ by $\tilde{L}(r|\eta|)$, since we do not have (4.2). Then the term II becomes

$$
\sup _{|\eta|>b|\xi|} \tilde{L}(r|\eta|)^{-1}\left|\hat{f}_{k}(\xi-\eta)\right| \int_{|\eta|>b|\xi|}\left|\hat{\chi}_{n}(\eta)\right| \tilde{L}(r|\eta|) d \eta
$$

and using (6.5) we can estimate this expression by a constant times

$$
\int_{|\eta|>b|\xi|}\left|\hat{\chi}_{n}(\eta)\right| \tilde{L}(r|\eta|) d \eta
$$

To estimate this integral we use (7.2) and obtain

$$
\begin{aligned}
I I \leq C \int_{|\eta|>b|\xi|}|\eta|^{-d-1}\left(B s_{n} /|\eta|\right)^{j} M_{j} d \eta=C\left(B s_{n} / b|\xi|\right)^{j} M_{j} \int_{|\eta|>1} \frac{d \eta}{|\eta|^{d+j+1}} \\
\leq C_{1}\left(B s_{n} / b|\xi|\right)^{j} M_{j}, \quad j \leq n,
\end{aligned}
$$

which completes the proof of Lemma 2 for ultradistributions.

To complete the proof of Theorem 2 we argue exactly as in Section 5 with the only difference that we must use the fact that $\chi_{n} \rightarrow \varphi$ in $\mathscr{D}_{L}(V)$ to be able to conclude $w_{n}\left(x_{d}\right) \rightarrow \int f \varphi d x^{\prime}$.

\section{References}

[B] Beurling, A., Quasianalyticity and general distributions, Lectures 4 and 5, A.M.S. Summer institute, Standford, 1961.

[Ba] Bang, Th., Om quasi-analy tiske funktioner, thesis, Copenhagen, 1946.

[Bj] Björck, G., Linear partial differential operators and generalized distributions, Arkiv for Mat., 6 (1966), 351-407.

[Bo1] Boman, J., A local vanishing theorem for distributions, C.R. Acad. Sci. Paris Sér. I, 315 (1992), 1231-1234.

[Bo2] - Uniqueness and non-uniqueness for microanalytic continuation of hyperfunctions, Preprint, 1996.

[C] Cohen, P., A simple proof of the Denjoy-Carleman theorem, Amer. Math. Monthly, 75 
(1968), 26-31.

[H1] Hörmander, L., Uniqueness theorems and wave front sets for solutions of linear differential equations with analytic coefficients, Comm. Pure Appl. Math., 24 (1971), 671-704.

[H2] - The analysis of linear partial differential operators 1, Springer-Verlag, Berlin, Heidelberg, and New York, 1983.

[H3] - Between distributions and hyperfunctions, in Colloque en l'honneur de Laurent Schwartz, Astérisque, 131 (1985), 89-106.

[H4] Hörmander, L., Remarks on Holmgren's uniqueness theorem, Ann. Inst. Fourier (Grenoble), 43 (1993), 1223-1251.

[Ka] Kaneko, A., Introduction to hyperfunctions, Kluwer Academic Publishers, Dordrecht, Boston, and London, 1988.

[Ko1] Komatsu, H., Ultradistributions, I. Structure theorems and a characterization, J. Fac. Sci. Univ. Tokyo Section IA, 20 (1973), 25-105.

[Ko2] - Ultradistributions, II. The kernel theorem and ultradistributions with support in a submanifold, J. Fac. Sci. Univ. Tokyo Section IA, 24 (1977), 607-628.

[Ko3] - Ultradistributions, III. Vector valued ultradistributions and the theory of kernels, J. Fac. Sci. Univ. Tokyo Section IA, 29 (1982), 653-717.

[dR] de Roever, J. W., Hyperfunctional singular support of ultradistributions, J. Fac. Sci. Univ. Tokyo Section IA, 31 (1984), 585-631.

[Rol] Roumieu, C., Sur quelques extensions de la notion de distribution, Ann. Sci. Ecole Norm. Sup., 77 Série 3 (1960), 41-121.

[Ro2] - Ultradistributions définies sur $R^{n}$ et sur certaines classes de variétés différentiables, J. d'Anal. Math., 10 (1962-63), 153-192.

[TT] Tanabe, S., and Takiguchi, T., A local vanishing theorem for ultradistributions with analy tic parameters, J. Fac. Sci. Univ. Tokyo Section IA, 40 (1993), 607-621. 
